\title{
Multiple-image super-resolution on mobile devices: an image warping approach
}

\author{
Neil Patrick Del Gallego* (iD and Joel llao
}

\begin{abstract}
This paper discusses a super-resolution (SR) system implemented on a mobile device. We utilized an Android device's camera to take successive shots and applied a classical multiple-image super-resolution (SR) technique that utilized a set of low-resolution (LR) images. Images taken from the mobile device are subjected to our proposed filtering scheme wherein images that have noticeable presence of blur are discarded to avoid outliers from affecting the produced high-resolution (HR) image. The remaining subset of images are subjected to non-local means denoising, then feature-matched against the first reference $L R$ image. Successive images are then aligned with respect to the first image via affine and perspective warping transformations. The LR images are then upsampled using bicubic interpolation. An $L_{2}$-norm minimization approach, which is essentially taking the pixel-wise mean of the aligned images, is performed to produce the final HR image.

Our study shows that our proposed method performs better than the bicubic interpolation, which makes its implementation in a mobile device quite feasible. We have also proven in our experiments that there are substantial differences from images captured using burst mode that can be utilized by an SR algorithm to create an HR image.
\end{abstract}

Keywords: Super-resolution, Mobile devices, Mean fusion, Image warping

\section{Introduction}

Image super-resolution (SR) refers to a class of techniques that produce a high-resolution (HR) image from observed low-resolution (LR) images. This LR image is initially upsampled to a higher desired resolution. Upsampling is normally done through image interpolation. However, high-frequency details are lost in image interpolation. Super-resolution attempts to recover the lost high-frequency details from an image after the initial upsampling step. This is referred to as the inversion of the image formation process [1], as seen in Fig. 1.

There are two known techniques for super-resolution, multiple-image SR and single-image SR. In this study, we explored the multiple-image SR technique on a mobile device by utilizing a set $(N=10)$ of observed LR images, that denote the same scene. We prove that multiple images captured using the burst mode feature of mobile devices can be utilized to create a super-resolved image. These input images undergo our proposed sharpness measure test in order to remove images with observable presence

*Correspondence: neil.delgallego@gmail.com

De La Salle University, Manila, Philippines of blur. The remaining subset of images are subjected to non-local means denoising and then image warping such that the LR images correspond to the same planar surface in respect of the reference LR image. Afterwards, all the observed LR images are merged to generate the HR image. Merging the LR images to generate the HR images is performed using an $L_{2}$-norm SR minimization approach [2]. This technique reverses the effects of aliasing and properly recovers the HR image.

Using this technique and a scaling factor of 4 , we evaluated the performance using real images taken from a mobile device and discussed the results. Our initial results using real images captured from a mobile device show noticeable improvement on the quality of HR images produced. We therefore present the following contributions:

1. Introduce a prototype application for performing multiple-image SR on a mobile device. Our approach has fast execution time and low memory footprint.

2. Proposed an automated ground-truth selection scheme to provide an unbiased assessment of our SR method.

3. Proved that capturing successive images using the burst mode feature of mobile devices contains 


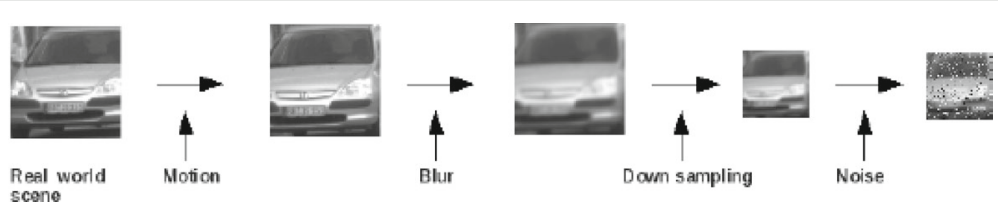

Fig. 1 Super-resolution involves the inversion of the image formation process [1]

sufficient information to tackle the super-resolution problem.

4. Tested and discussed results of real images taken from a mobile device. From our review of related work, only the work of Chu (2013) also tested their proposed algorithm on real images from a mobile device [3].

5. Proposed a filtering scheme that removes unwanted images prior to performing the SR algorithm to improve the results.

\section{Related work}

This section discusses the related work of this research. The research of Chu (2013) is closely related to our study wherein they address the super-resolution problem using the Shift-Add fusion technique [3, 4]. The initial HR image is further refined using a customized image regularization scheme tailored for mobile devices. Our approach is therefore influenced by their study. The related work section is divided accordingly, as follows: solving the downsampling problem, denoising, deblurring, identifying presence of motion, and image regularization. Lastly, we discuss how SR algorithms are assessed.

\subsection{Overview}

Images that are downsampled lose actual pixel values from its HR counterpart. Recent works in super-resolution specialized in solving the downsampling problem and assumed that images do not have any blur or motion present. The method called Adjusted Anchored Neighborhood Regression proposed by Timofte et al. (2015) managed to produce HR images with acceptable quality provided that the LR image counterparts are sharp
$[5,6]$. According to the authors, SR is mainly concerned with upscaling the image without losing its sharpness. In the context of images taken from mobile devices, noise, blur and motion affect the quality of the image produced. Thus, it is necessary to apply some techniques to attempt to solve the SR problem when other degradation factors are present. However, it is known that removing these unwanted effects are computationally heavy. In fact, each of these areas can be considered as a research problem on its own. There are separate research areas in denoising [7-9], and in deblurring (tackling out of focus or motion blur) [10-12]. We highlight the methods presented on these areas in the in the succeeding subsections.

\subsection{Solving the downsampling problem}

The simplest approach to solve the downsampling problem is to perform interpolation. However, image interpolation does not recover high-frequency details in the HR image. Recent works in SR deal with single images and apply machine learning or regression mechanisms to associate an LR image patch to an HR image patch counterpart. The algorithm proposed by Timofte et al. (2015) is the fastest known method under this approach [5]. While it is considerably fast, it does not tackle other degradation factors as identified by Mitzel et al. (2009) and presented in Fig. 1 [1].

In the context of multiple-image SR, Shift-Add fusion was proposed by Simpkins and Stevenson (2012) to solve the downsampling problem which is a fundamental operation of the multiple-image SR technique [4]. Given an LR image (or a set of LR images), recovering the missing pixels on the HR grid is done based from the pixel values found
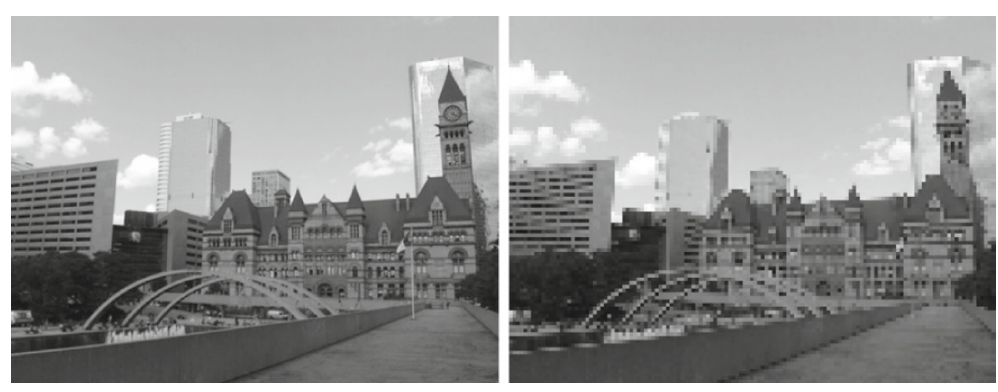

Fig. 2 An HR ground-truth image (left), and its LR observation (right) [4] 


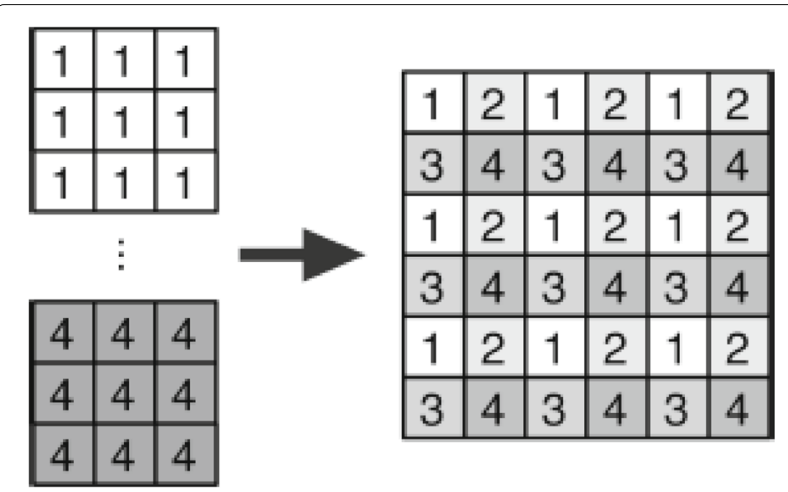

Fig. 3 Under known motion estimates, each pixel in $L R 1$. . LR4 corresponds to a specific location in the HR image [4]

on the LR image set. Shift-Add fusion attempts to reverse the effects of aliasing.

An LR image shows signs of aliasing, which is a distortion or artifact caused by different sampling rates used to produce digital images [13]. It is a fundamental problem that affects the quality of digital images. Refer to Fig. 2 for discussion.

The left image represents the HR ground-truth image and a simulated LR observation on the right is created. A magnification factor $S$ of 3 is used to simulate the LR observation. Notice the artifacts introduced caused by aliasing. The aliasing effect is what makes digital images visually unappealing. Different aliased images make it possible to recover an HR image [4]. Works of Chu (2013), Peleg, Keren, and Schweitzer (1987), Huang and Tsay (1984) revolved around this idea [3, 14, 15].

The theoretical basis for SR is best explained in the frequency domain $[4,16]$. However, most SR works are performed on the spatial domain and it is here where intuitive techniques are proposed [16]. The concept of Shift-Add fusion is simple. It is known that each LR observation contains spatial shifts [4]. If motion estimates properly model the spatial shifts found in the set of LR observations (either explicitly stated or an estimation procedure is performed), then each LR pixel corresponds to a certain missing pixel in the HR image as seen in Fig. 3. We therefore, properly recover the HR image.

\subsection{Denoising}

Images captured from the mobile device contains unwanted noise. Simple noise removal can be performed by filtering. However, denoising methods perform better than basic filtering such as median filter. The study of Yoshida, Murakami and Ikehara (2013) tackled the SR problem based on non-local means denoising $[8,17]$. We also applied the technique of Buades et al. (2011). The difference in our technique is that image denoising is performed prior to upsampling because we observed that performing denoising after upsampling did not yield observable differences. This makes the denoising algorithm execute faster.

\subsection{Deblurring}

According to Simpkins and Stevenson (2012), the presence of blur in the input LR images will make it theoretically impossible to recover the high-frequency details via Shift-Add fusion approach [4]. Deblurring itself is a dense research area. We only discuss the work of Chu (2013) in this subsection. The work of Chu (2013) managed to simplify this problem by considering linear motion of shaken angle and shaken length. The small aperture of most cameras installed on mobile devices lead to short focal length and deep depth-of-field (DOF) of the camera [3].

To prove this statement, Chu (2013) conducted an experiment by taking images with induced camera shake from a mobile device, and from a digital camera. Figure 4 , taken from the discussion of Chu (2013), shows that the motion blur induced by shaking the mobile device is linear, where a linear blur kernel is a slanting dotted line. The digital camera shows a non-linear motion blur. Therefore,
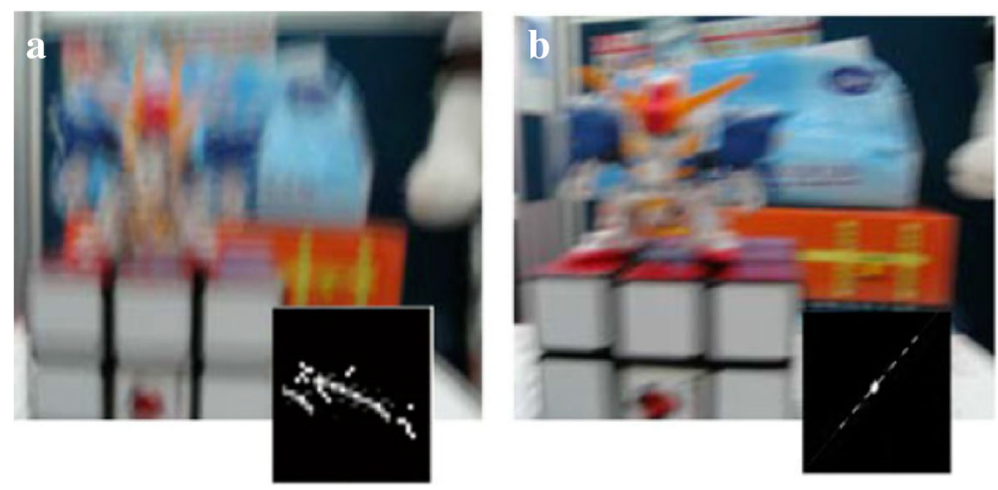

Fig. 4 a Motion blur induced by capturing from a digital camera (Nikon D50). b Motion blur induced by capturing from a mobile device (HTC Desire HD) [3] 


\begin{tabular}{|l|l|l|l|l|l|}
\hline 1 & 2 & 1 & 2 & 1 & 2 \\
\hline$?$ & 4 & 3 & 4 & 3 & $?$ \\
\hline 1 & 2 & 1 & $?$ & 1 & 2 \\
\hline 3 & 4 & $?$ & $?$ & 3 & 4 \\
\hline 1 & $?$ & 1 & 2 & $?$ & $?$ \\
\hline 3 & $?$ & 3 & 4 & $?$ & $?$ \\
\hline
\end{tabular}

HR image

Fig. 5 The pixel grid with question marks denote missing pixels not properly determined by the Shift-Add fusion technique

they conclude that linear motion deblurring is applicable for mobile devices [3].

\subsection{Identifying presence of motion}

Motion estimation is essential in identifying the assignment of pixel values in the HR grid for the Shift-Add fusion technique. It is also used for image registration. The image warping technique, which was used in our study, is used to properly align the images of the same scene. Other methods involve the use of optic flow fields, as shown in the study of Chu (2013). Dense optical flow like the LucasKanade method is not appropriate for mobile devices due to its slow execution time.

Pixel-level motion displacement was formulated by Farneback (2003) which may seem ideal for the Shift-Add fusion technique [18]. We attempted to use this technique but our initial results show that image warping performs better than using the method proposed by Farneback (2003).

\subsection{Image regularization}

Regularization introduces additional information to a function in order to solve an ill-posed problem or avoid overfitting. Regularization is also applied to the SR problem. The concept of image regularization attempts to further refine or recover the HR image that is otherwise not covered properly by the Shift-Add fusion technique [4]. Suppose that there are still missing pixel values in the HR image. This is normally caused by undersampled or missing observed pixels in the LR set, or the values are simply not reliable. Refer to Fig. 5 for this discussion. The question marks denote missing pixels that were not recovered using the Shift-Add fusion technique. Shift-Add fusion works together with image regularization such that when the Shift-Add fusion fails to recover all supposed pixel values in the HR image, the image regularization model would fulfill the remaining work.

Image regularization is also called regularized image reconstruction which is referred to as "inpainting" in other related works $[4,19]$. Image regularization works by attempting to determine the missing pixel values, denoted by an image mask, as seen in Fig. 6 .

\subsection{Assessment of SR techniques}

Performance assessments of SR algorithms use groundtruth HR images that are downsampled to produce the corresponding LR images. Some works introduced noise or blur operators to further distort the LR image [20-22]. This LR image is used as input to the system and SR is performed to produce a super-resolved HR image. The super-resolved HR image is compared with the groundtruth image to assess how much details were actually recovered. Common measurements are peak signal-tonoise ratio (PSNR), structural image similarity (SSIM), root mean squared error (RMSE), and pixel-based average error [16].

\section{Methodology and system implementation}

This section discusses the implementation of our proposed system, which is heavily derived from the concepts discussed in Section 2. A prototype was developed, which is capable of performing multiple-image SR on Android devices. These devices can be set to capture photos in burst mode, wherein a series of shots are taken successively. This feature was introduced on Android 5.0. Comparable results were observed using a scaling factor, $S=4$, rather than $S=2$. Thus, we only discuss the results using
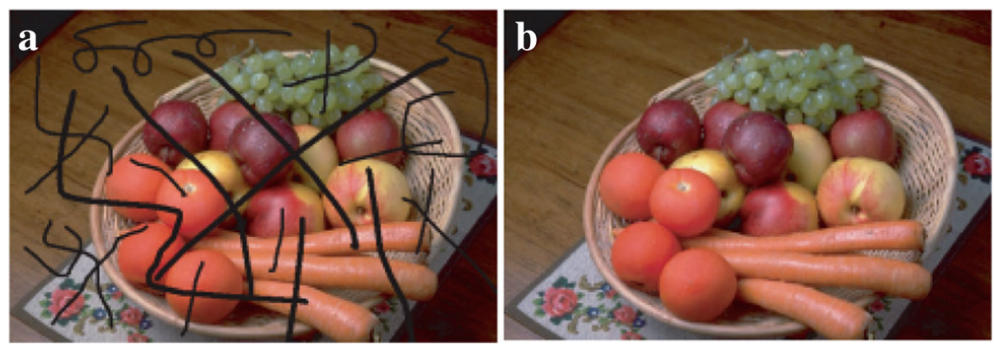

Fig. 6 Given an input image (a) with black pen marks, inpainting or regularization is done to recover the missing pixels, seen in image (b) [19] 
this value on this paper by measuring the PSNR, RMSE, and SSIM of our super-resolved image.

\subsection{Measuring the differences between images}

Multiple-image SR technique will be applicable if each LR image present in a given set contains unique information. To validate this claim, we performed an experiment to measure the differences of each LR image against the first LR image taken. We performed this test prior to using the images as input for our system. We used the SSIM (structural image similarity) measure as our preferred metric to quantify the differences present. In this case, a low SSIM indicates noticeable differences when compared to the first LR image. On all our seven test images, a mean SSIM of 0.6004 were obtained, which is favorable for our study. Table 1 shows the average SSIM per image. Figure 7 shows the best case wherein differences are observably present in the LR image set.

\subsection{Assessment technique and ground-truth image selection}

Our methodology for assessing our SR technique is similar to related works (discussed in Section 2.7) [16]. However, we observed from multiple-image SR works $[1,2,23]$ that no objective method were discussed regarding ground-truth selection. The study of Chu (2013) used a different camera and used the images taken from it as ground-truth [3]. Single-image SR works commonly used benchmark images from public dataset as ground-truth and test images were downsampled from it $[5,20,21,24]$. Few research works on SR, however, utilize objective performance measures such as PSNR, RMSE, and SSIM. Only the visual quality are assessed [1, 2, 23]. From our review of related work, the work of $\mathrm{Chu}$ (2013) managed to tackle their results using the SSIM measure [3].

With no concrete standard on related literature, we therefore present our proposed assessment technique and ground-truth image selection for multiple-image SR tackled on a mobile device. We can choose to manually pick our own ground-truth image from the image set but doing so will introduce bias on our tests. Normally, ground-truth images should be clear and blur should be minimal (or none at all). With this reasoning, we formulated an automated approach wherein the best image from a given set is selected as the groundtruth. The selected image is then removed from the input set and then the input set is forwarded to our SR algorithm to formulate an approximate HR image as close to the ground-truth image selected. The automated ground-truth image selection approach is discussed in the "Appendix".

Our assessment technique is illustrated in Fig. 8. The input LR images, without the ground-truth image, are downsampled prior to feeding it into the system. The
Table 1 Images tested and their average difference measured by SSIM. Lower value indicates greater difference from the first LR image

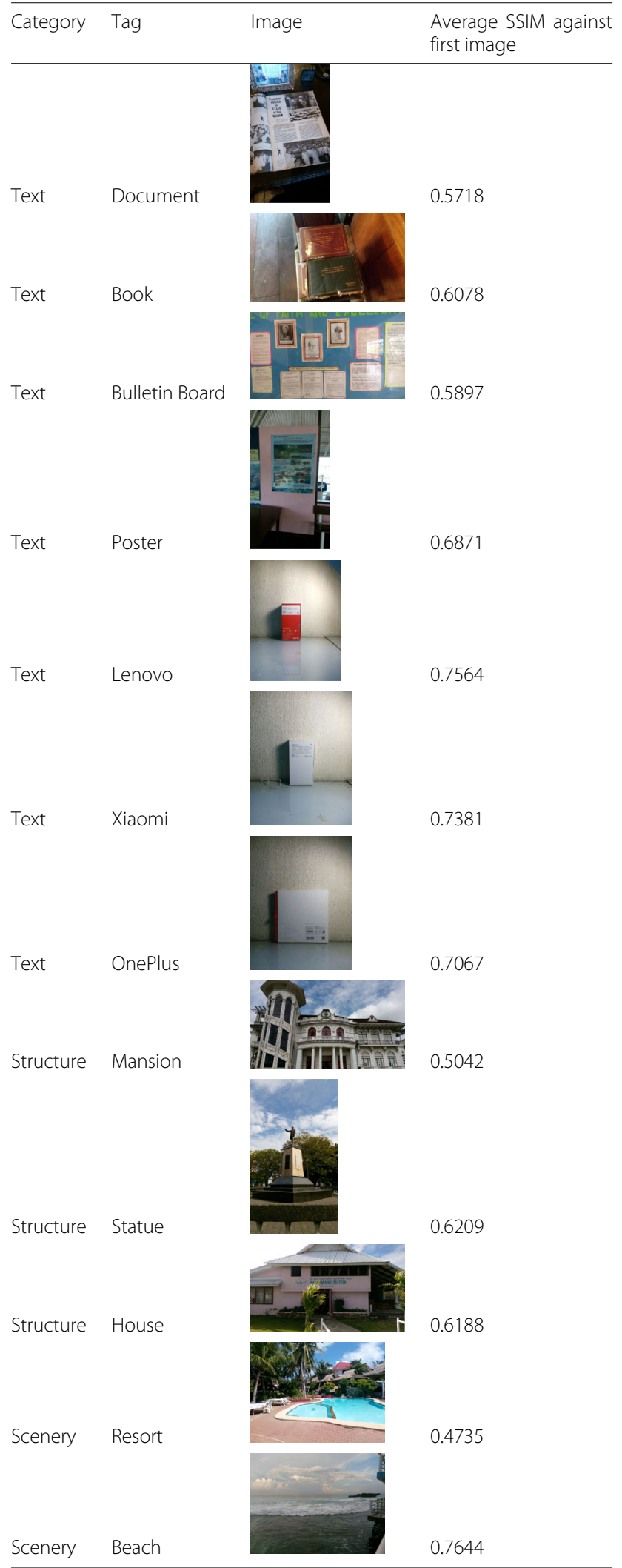




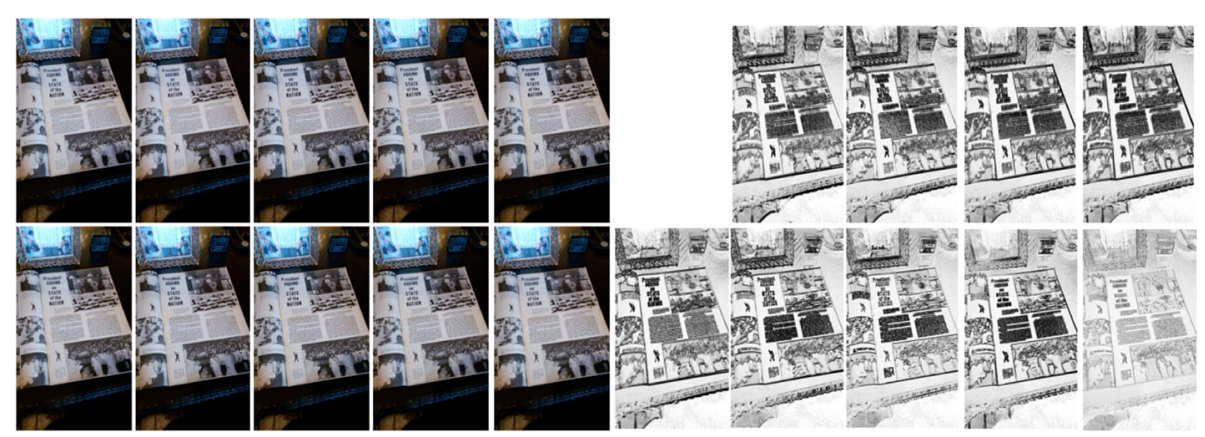

Fig. 7 An example of SSIM map for the document image. Darker values denote greater differences from the first LR image

output HR image produced is compared with the groundtruth image using PSNR, RMSE, and SSIM.

\subsection{System architecture}

Our system accepts a set of LR images wherein the first LR image serves as the base LR image. The LR images undergo a feature-selection scheme wherein LR images that are affected by blur are removed. The selected LR images are feature-matched, and affine transformation and perspective warping were performed to the images with respect to the base LR image. We then performed an "interpolation and fusion" approach [23] and performed averaging to combine the images and produce the HR image. This is essentially taking the pixel-wise mean that has been proven by Farsiu et al. (2004) as a multiple-image $S R$ method using $L_{2}$-norm minimization [2]. We discuss this technique further in relation to the diagram shown in Fig. 9. Specific details for each major step in the system architecture are further broken down into subsections in this paper.

Initial input LR images undergo our automated sharpness measure test. This approach is similar to our ground-truth image selection scheme briefly mentioned in Section 3.2. Details about this test is discussed in "Appendix". The goal of this test is to measure the sharpness of all the input images and compute the mean. Images with sharpness values that fall below the mean are discarded as this will just degrade the overall result of the produced HR image.
Therefore, given a set of filtered LR images, $\left\{L_{0} \ldots L_{N}\right\}$, $L_{0}$ is upsampled to produce nearest-neighbor interpolated HR image, and a bicubic interpolated HR image for comparison. Using the LR image set, $\left\{L_{0} \ldots L_{N}\right\}$, the images undergo the following steps: non-local means denoising, affine warping, perspective warping, bicubic interpolation, then mean fusion.

\subsection{Non-local means denoising}

Filtered images $\left\{L_{0} \ldots L_{N}\right\}$ are subjected to non-local means (NLM) denoising method [8]. Denoising is to prevent unwanted artifacts in the input images from manifesting in the HR image. The intention of NLM denoising is to mitigate that problem. NLM denoising was also derived from the study of Protter and Elad (2009) which formulated a super-resolution reconstruction method based from NLM and also from Yoshida, Murakami and Ikehara (2013) which proposed a different SR approach based from self-similarity and NLM $[17,25]$. The difference in our proposed method is to directly apply NLM denoising on the luminance channel of the image and perform it as a separate task instead of performing super-resolution at the same time. This process is also performed prior to performing our proposed SR method unlike other works.

\subsection{Feature matching, affine and perspective warping}

The subset, $\left\{L_{1} \ldots L_{N}\right\}$, is subjected to image warping using matched features to $L_{0}$. Feature-matching is done using

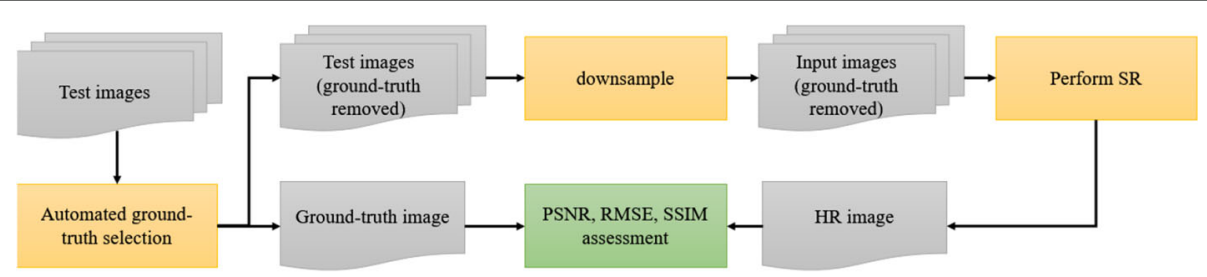

Fig. 8 Methodology of assessing our multiple-image SR technique 


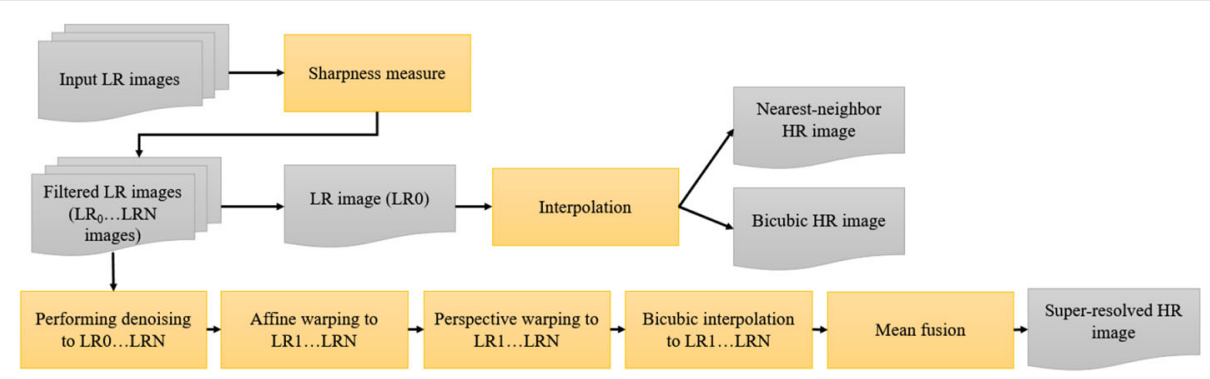

Fig. 9 Multiple-image SR system architecture

the ORB descriptor [26], which was introduced by Rublee (2011). Once the features have been found on images in $\left\{L_{1} \ldots L_{N}\right\}$, the keypoints are matched to $L_{0}$ using a bruteforce approach and refined by RANSAC. This method is seen in Fig. 10.

Once the features have been matched, $\left\{L_{1} \ldots L_{N}\right\}$ are warped, by identifying the affine and perspective transformation using $L_{0}$ as the source point, to produce $\left\{W_{1} \ldots W_{N}\right\}$.

This concept is illustrated in Fig. 11 wherein the warped images correspond to a similar point in a planar surface. When the images are merged, it is supposed to blend properly as one combined image.

\subsection{Combining warped images to form the HR}

Once the warped images have been processed, the warped images are combined using mean fusion as illustrated in Fig. 12. Let $W_{0}=L_{0}$, so we now have the set $\left\{W_{0} \ldots W_{N}\right\}$. $\left\{\hat{W}_{0} \ldots \hat{W}_{N}\right\}$ can therefore be derived from $\left\{W_{0} \ldots W_{N}\right\}$ by simply upsampling the images individually. For this study, we simply performed bicubic interpolation on the images so no missing pixel values needs to be regularized. We then performed mean fusion approach to combine the images which is formally a multiple-image SR method using $L_{2}$-norm minimization [2].

\subsection{Other details on implementation}

We implemented a prototype on an Android device and used the camera burst mode to take 10 images to be

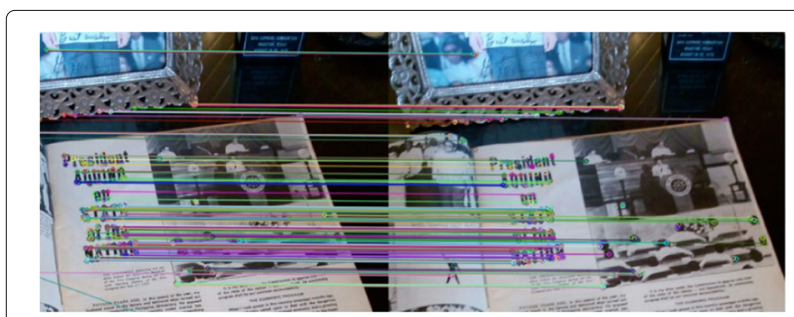

Fig. 10 Feature matching using ORB descriptor. Points are matched using brute-force with simple distance thresholding and further refined by RANSAC fed into the SR system. The images enter the modules described in the previous subsections as a whole set. Individual images are processed in each module in a sequential manner. Like other SR works, we only performed our proposed SR method on the luminance $(\mathrm{Y})$ channel of the images and simply performed bicubic interpolation on the chroma channels (UV).

\section{Results and observations}

Using the methodology mentioned in Section 3, results are discussed here as well as our visual evaluations of the test images. We tested the following on the following test images summarized in Table 1. A visual comparison is provided against the ground-truth image as well as numerical metrics such as PSNR (peak signal to noise ratio), RMSE (root mean squared error), and SSIM (structural image similarity). Note that the scaling factor used in the experiments is 4 .

Results using PSNR, RMSE, and SSIM are provided in Tables 2, 3, and 4, respectively. The structure of this section is to discuss different cases where the system performs best, and cases where the system does not perform favorably.

\subsection{Test cases using images with text}

The proposed system works best with images that contain texts, as edges are properly recovered. Two examples, using Text_Newspaper and Text_BookCover, are shown in Fig. 13. Although the SSIM difference for the document is minimal from the bicubic image (0.0070 difference), the texts have been properly recovered by our proposed SR method.

\subsection{Out-of-focus images}

The system has been observed to perform well on properly recovering high-frequency details when the subject is out of focus. Due to the capability of capturing multiple images of the same subject, and the effectiveness of our automated sharpness measure test, the system manages to select good images for creating an HR image. In the case of mobile devices, a subject becomes out of focus if the camera did not properly lock focus on the subject. Consider 


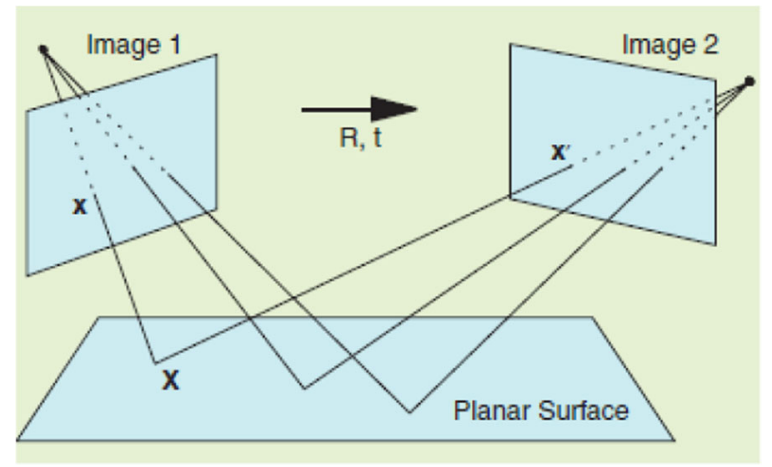

Fig. 11 How images should correspond to a certain point in a planar surface [27]

the example shown in Fig. 14. Image subjects are out-offocus when the user has difficulty selecting a focus point for the camera, the camera auto-focus mode is disabled, or there is not enough time to focus on the subject during image capture (i.e., user is in a moving vehicle, attempting to capture a fixed subject outside).

Figure 15 shows the results of the system under such cases. Images for Text_Lenovo and Text_Xiaomi image sets have been captured with auto-focus mode disabled, which should produce the same issue presented in image in Fig. 14a. The ground-truth image has been captured separately with focus mode enabled and locked on the subject properly. Despite this limitation, our proposed system manages to recover some of the texts, which makes it more readable than the results obtained from interpolation methods. Numerically, the results are also better than bicubic interpolation (0.0223 improvement). Thus, for such cases wherein focusing on the subject is difficult, our proposed system is recommendable.

\subsection{Tolerance to noise}

The system performs well in reducing the level of noise in the final HR image, due to the denoising step and the mean fusion process. For this experiment, the sample image set, One+_Noise, was used and random noise was added to the input images, as seen in ImageJ in Fig. 16. The denoising step and the mean fusion process noticeably reduce image noise and properly restore high-frequency information, as observed in the visual comparison in Fig. 16. The SSIM of our proposed system is also significantly higher $(0.1975$ difference), than the bicubic interpolation method.

\subsection{Outdoor text images with proper focus}

Text_BulletinBoard and Text_Poster image sets are taken from outdoors, with proper focus on the image subject. The focus point was locked on the subject while acquiring the images, which is the opposite of the experiment discussed in Section 4.2. The system did not perform as intended on these image sets. The HR images produced by

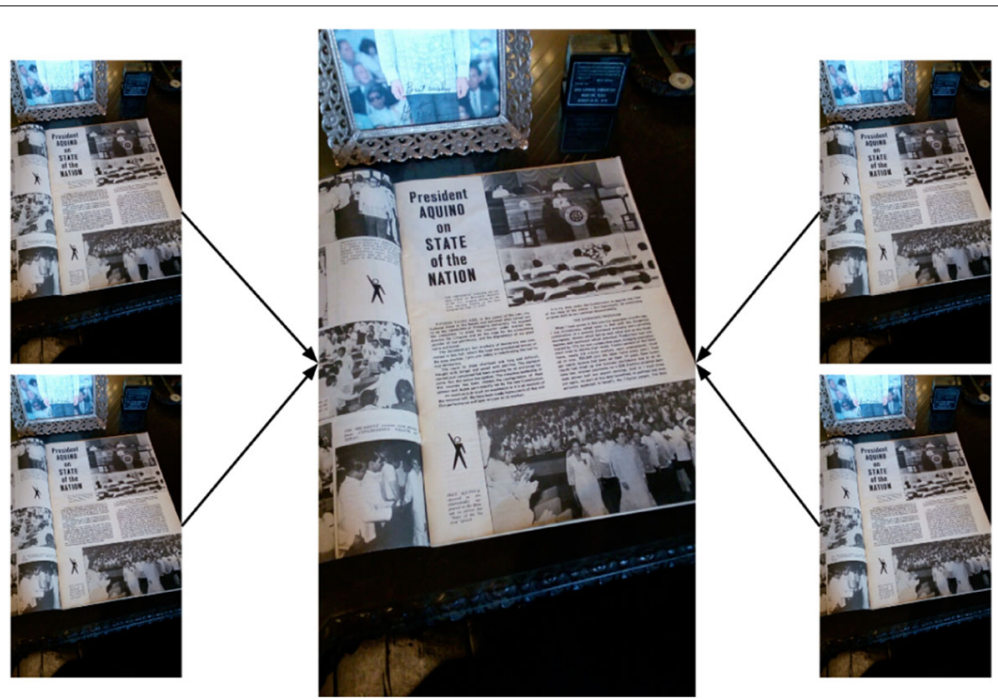

Fig. 12 Given 4 HR warped images, it is combined by mean fusion 
Table 2 PSNR results for images. Higher value is better. Highest/lowest values italicized

\begin{tabular}{lllllll}
\hline PSNR measurement & Text_Newspaper & Text_BookCover & Text_Bulletin Board & Structure_Mansion & Structure_Statue & Structure_House \\
Nearest-neighbor & 16.4686 & 21.9638 & 18.6582 & 17.1456 & 17.9367 & 19.2184 \\
Bicubic & 16.4538 & 21.9569 & 18.6621 & 17.1563 & 17.9546 & 19.2167 \\
SR method & 16.2568 & 22.3743 & 18.9034 & 17.3134 & 19.5573 & 20.0862 \\
PSNR measurement & Text_Poster & Text_Lenovo Specs & Text_Xiaomi Specs & Scenery_Resort & Scenery_Beach & One+_Noise \\
Nearest-neighbor & 18.3682 & 30.1958 & 25.6326 & 21.6374 & 24.648 & 23.487 \\
Bicubic & 18.3741 & 30.5818 & 25.6167 & 21.9283 & 24.686 & 23.8614 \\
SR method & 18.5287 & 32.9122 & 27.5933 & 22.9866 & 26.7942 & 24.8408 \\
\hline
\end{tabular}

our SR method for the announcement board and poster test image can be observed as soft and washed out, and no high-frequency details can be observed. Judging it visually in Fig. 17, our SR method did not manage to recover enough high-frequency details to make the texts readable. The SSIM results of both images are, otherwise, higher than the bicubic interpolation (0.0206 and 0.0178 difference). The denoising step performed as preprocessing may have some high-frequency details filtered as false positives which have affected the overall result of the HR image. Thus, it is observed that the system may not be beneficial on cases wherein capture conditions are already ideal (i.e., camera has successfully locked focus on the subject, user's hand is steady).

\subsection{Test cases on structures}

Structure_Statue, Structure_Mansion, and Scenery_Resort have man-made structures as the subject. The system does not properly model the edges of the structures and the textures that should ideally be present on the HR image. The denoising step may have removed some high-frequency details that can be utilized for HR reconstruction. While our SR method performed slightly better than the bicubic interpolation (0.0148, 0.0382, and 0.0364 improvement), the results of images in Fig. 18 show that the edges are not observably better than the bicubic interpolation method. The result of the system in Structure_Statue has little visual difference from the bicubic interpolated image. The edges and textures shown in the result of
Structure_Mansion appear soft and washed out. The pool tiles in Scenery_Resort were not present on the HR image.

\subsection{Test cases with presence of motion}

While the SSIM result of the HR image in Structure_Mansion and Scenery_Beach are better than the bicubic image (0.0262 improvement), the system does not perform well on moving objects. Referring to Fig. 19, notice that our SR method introduces a ghosting effect on the leaves that are moving. It is also observed in Fig. 20 where the movement of the waves caused unwanted ghosting effects. Considering this case, it can be taken as a factor for improvement of our SR method. A similar case, discussed in Section 4.5, is also observed in Fig. 20, wherein the textures and the edges of the rock were not fully recovered by the system.

\section{Conclusions}

In this paper, we developed a prototype application that utilizes a mobile device's camera to take pictures and applied a classical multiple-image SR technique that utilized a set of LR images. In the pre-processing stage, the input LR images undergo a sharpness measure test wherein images that are blurred are discarded from the set. We then performed an "interpolation and fusion" approach and performed averaging to combine the images and produced the HR image. This is essentially taking the pixel-wise mean of the images that has been proven by Farsiu et al. (2004) as a multiple-image SR method using

Table 3 RMSE results for images. Lower value is better. Highest/lowest values italicized

\begin{tabular}{lllllll}
\hline RMSE measurement & Text_Newspaper & Text_BookCover & Text_Bulletin Board & Structure_Mansion & Structure_Statue & Structure_House \\
Nearest-neighbor & 7.4428 & 5.4065 & 7.4906 & 7.4378 & 7.5256 & 6.2547 \\
Bicubic & 7.4381 & 5.3942 & 7.5087 & 7.4800 & 7.5220 & 6.5675 \\
SR method & 7.3347 & 5.1708 & 7.4939 & 7.4433 & 6.7570 & 6.5488 \\
RMSE measurement & Text_Poster & Text_Lenovo Specs & Text_Xiaomi Specs & Scenery_Resort & Scenery_Beach & One+_Noise \\
Nearest-neighbor & 7.5445 & 3.8570 & 5.4641 & 5.9532 & 5.3968 & 2.5629 \\
Bicubic & 7.5482 & 3.7870 & 5.4610 & 5.9489 & 5.3838 & 2.3919 \\
SR method & 7.3399 & 3.0546 & 4.1848 & 5.7057 & 4.9253 & 1.6789 \\
\hline
\end{tabular}


Table 4 SSIM results for images. Value closer to 1.0 indicates high similarity to the ground-truth. Highest/lowest values italicized

\begin{tabular}{llllllc}
\hline SSIM measurement & Text_Newspaper & Text_BookCover & Text_Bulletin Board & Structure_Mansion & Structure_Statue & Structure_House \\
Nearest-neighbor & 0.6463 & 0.7397 & 0.6721 & 0.5797 & 0.6631 & 0.7127 \\
Bicubic & 0.6613 & 0.7531 & 0.6892 & 0.5955 & 0.6772 & 0.7355 \\
SR method & 0.6683 & 0.7695 & 0.7098 & 0.6103 & 0.7154 & 0.7617 \\
SSIM measurement & Text_Poster & Text_Lenovo Specs & Text_Xiaomi Specs & Scenery_Resort & Scenery_Beach & One+_Noise \\
Nearest-neighbor & 0.7342 & 0.8367 & 0.7748 & 0.6316 & 0.8079 & 0.5199 \\
Bicubic & 0.7452 & 0.8567 & 0.7877 & 0.6563 & 0.8241 & 0.6155 \\
SR method & 0.7630 & 0.8790 & 0.8103 & 0.6945 & 0.8605 & 0.8130 \\
\hline
\end{tabular}

$L_{2}$-norm minimization [2]. Aside from our SR method, we also introduced a scheme on automating the selection of ground-truth images for unbiased assessments.

As seen from the numerical results, our SR method performed justifiably better than interpolation methods in terms of PSNR, RMSE, and SSIM. It has been observed that the system performs best on text images, particularly those captured indoors. The edges of the texts have been properly recovered by the system, making the texts more readable than interpolation methods. The system is particularly suitable for dealing with images that are out-offocus. Our automated sharpness measure test proves to be effective by managing to select good images for recovering the HR scene despite having out-of-focus samples. Our proposed method becomes ideal when capturing images where focusing on the subject becomes difficult (i.e., user is in a moving vehicle, attempting to capture a fixed subject outside. The user's hands are not steady). Vice-versa, if the capture conditions are already ideal (i.e., camera has properly locked focus on the subject, user's hands are steady), our system does not perform as intended. Thus, a simple bicubic interpolation method may be sufficient to properly produce an HR image from a single LR image with minimal noise and blur.

Should noise be present on the captured images, our denoising step and mean fusion noticeably reduce image noise in the output HR image and properly restore highfrequency information. However, due to the nature of the

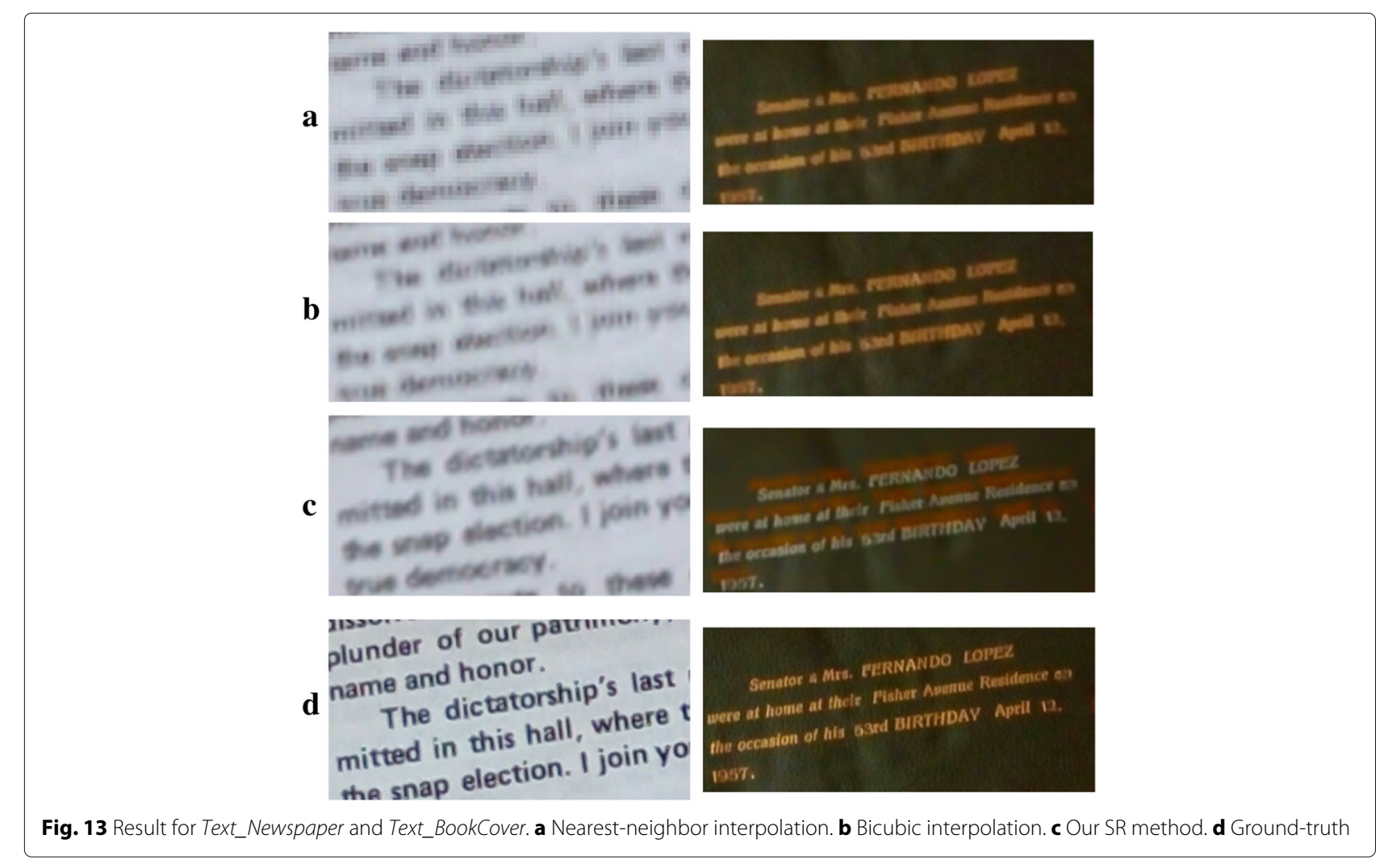




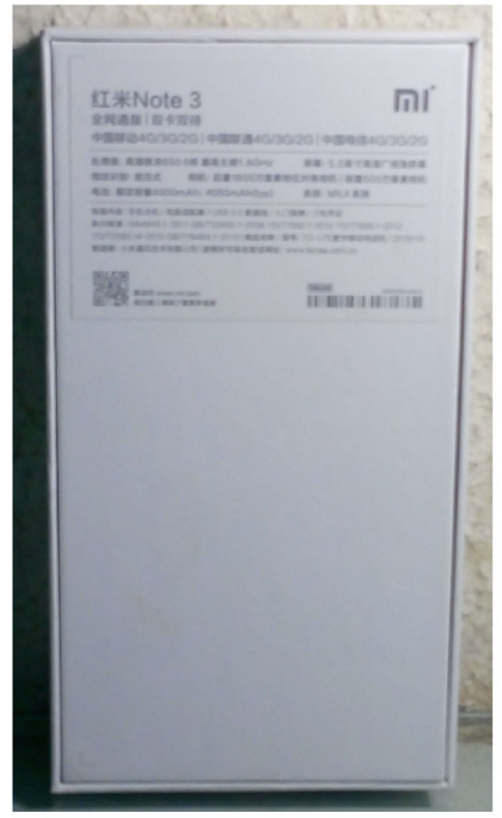

$\mathbf{a}$

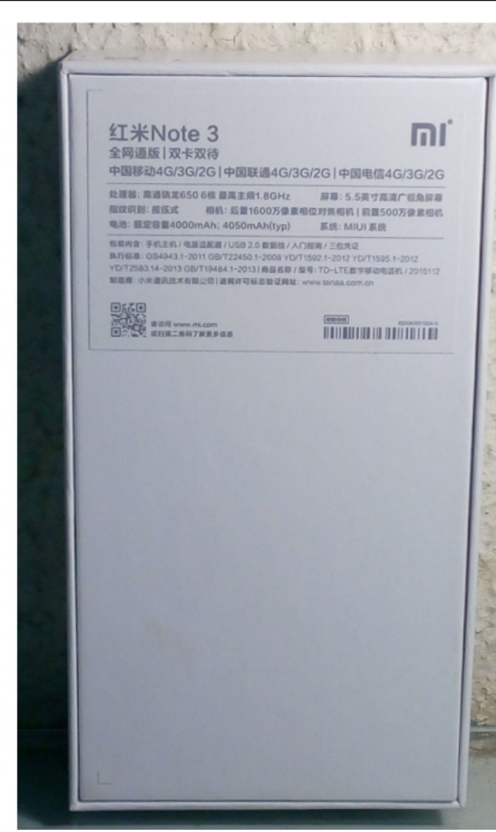

b

Fig. 14 An out-of-focus image example. The subject becomes out of focus, as seen in image $\mathbf{a}$, if the camera did not properly lock its focus. Image $\mathbf{b}$ is noticeably clearer when the camera successfully locked its focus on the subject

mean fusion method, our system is not recommendable for capturing images with presence of subject motion. This introduces ghosting effects and unwanted artifacts that occur when the HR image is produced.

It is also observed that the system does not perform acceptably on images captured outdoors and images with man-made structures. This involves architecture, buildings, sceneries, and environment, where tiles, repeating patterns, and natural textures are present. Edges are not properly recovered and textures are incorrectly represented or removed.

Overall, our proposed method is fast as it only involves accumulating the pixel-wise values of the images and then taking the mean. Furthermore, not all LR images from a given set are used because some images that did not pass our sharpness measure test are discarded.

The accuracy of the mean fusion approach is highly dependent on the accuracy of alignment of the LR images. $\mathbf{a}$

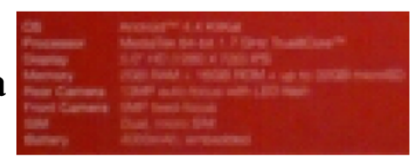

b

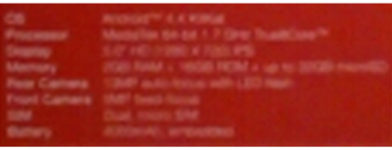

c

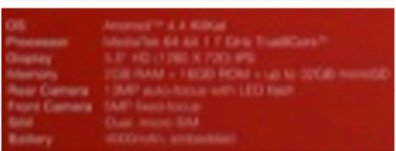

d

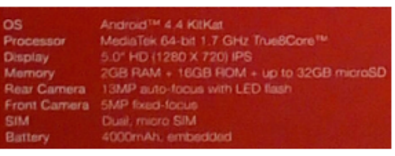

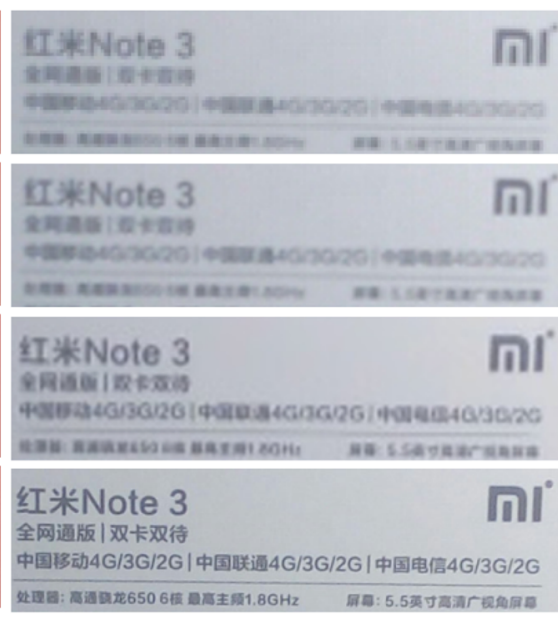

Fig. 15 Result for Text_Lenovo and Text_Xiaomi. a Nearest-neighbor interpolation. b Bicubic interpolation. c Our SR method. d Ground-truth 


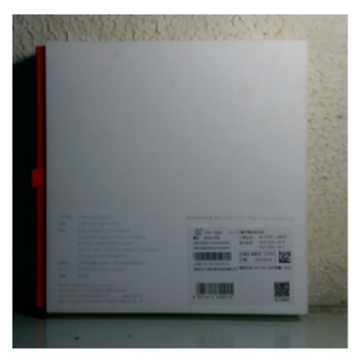

i

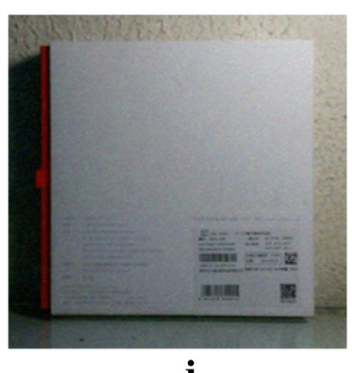

j

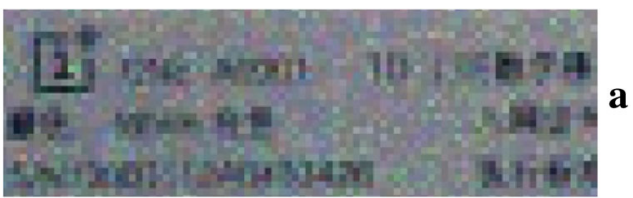

$\mathbf{a}$

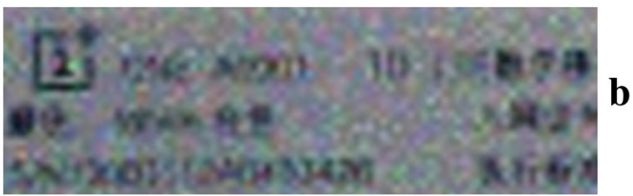

b
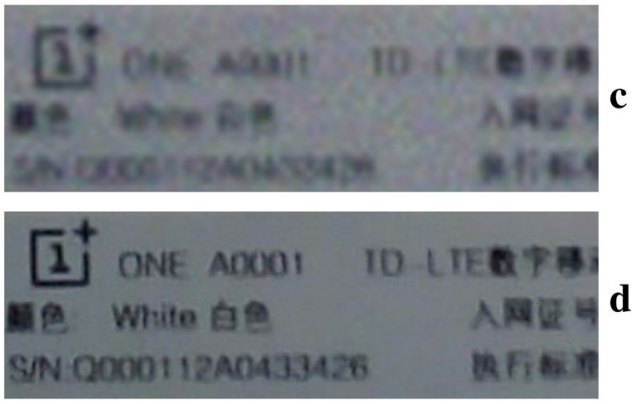

Fig. 16 Result for One+_Noise. a Nearest-neighbor interpolation. b Bicubic interpolation. c Our SR method. d Ground-truth. i Sample input image. j Input image with random noise added

Misalignments caused by moving objects have a ghosting effect on the produced HR image. While our work is preliminary, we see some feasibility on further improving our results and a clear direction on our study. First, we will strongly consider transitioning into an $L_{1}$-norm minimization SR because the study of Farsiu et al. (2004) showed that it is more robust and highlights edges more clearly [2]. Thus, from a pixel-wise mean, $L_{1}$-norm minimization is essentially performing a pixel-wise median approach. However, due to the nature of computing the $\mathbf{a}$
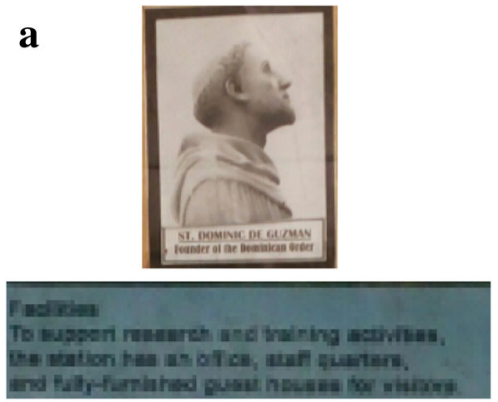

c
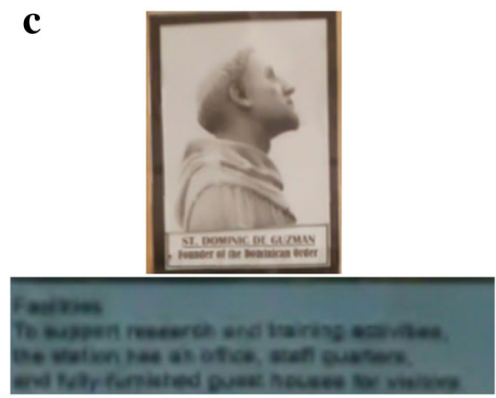

b
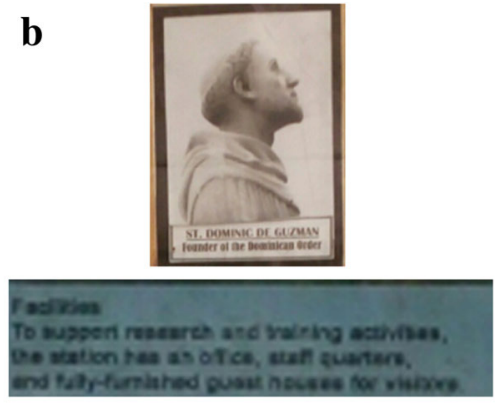

d

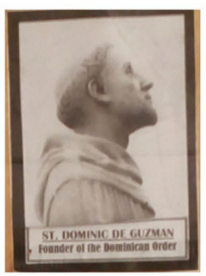

Facilities

To support rosearch and training activities,

the station has an office, staff quartere.

and fully-furniahed guest houses for visi

Fig. 17 Result for Text_BulletinBoard and Text_Poster. a Nearest-neighbor interpolation. b Bicubic interpolation. c Our SR method. d Ground-truth 

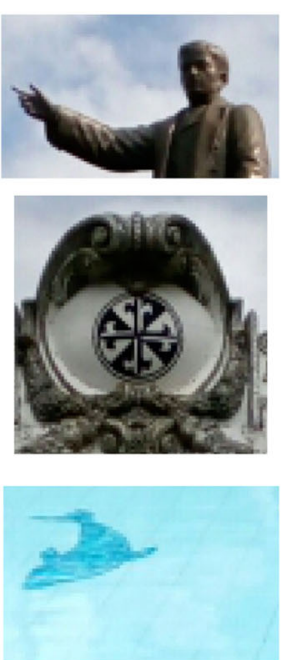

a
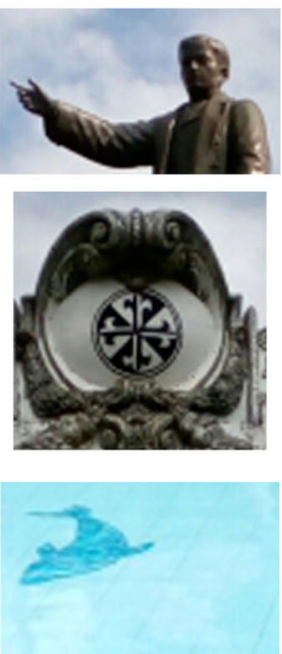

b
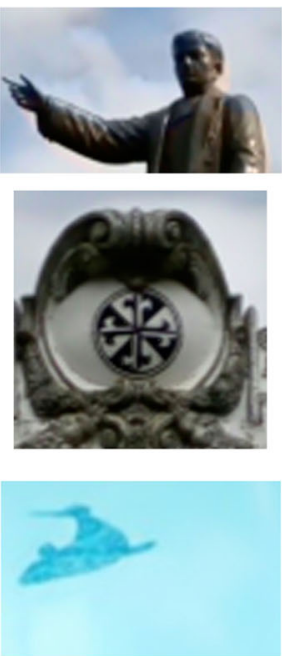

c
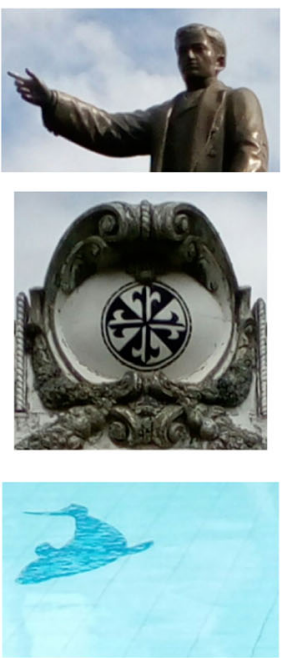

d

Fig. 18 Result for Structure_Statue (top), Structure_Mansion (middle), and Scenery_Resort (bottom). a Nearest-neighbor interpolation. b Bicubic interpolation. c Our SR method. d Ground-truth. The result of Structure_Statue do not have any observable difference from the bicubic interpolation. The result of Structure_Mansion appear soft and washed out. Pool tiles were not present in the output of Scenery_Resort

median (sorting then taking the middle value), doing median fusion will greatly affect the processing time. Thus, a clear improvement is to further refine our existing fusion approach to be as close as the accuracy imposed by median fusion, without greatly affecting the processing time.

Another room for improvement is to formally introduce an image regularization scheme such that high-frequency details are properly recovered while minimizing noise. Performing non-local means denoising removes potential high-frequency details that can be utilized for HR reconstruction. Therefore, this approach needs further validation. The next major step for this research after we have proven that our SR algorithm have more favorable results, is to tackle time and space complexity for performing super-resolution on mobile devices. This involves optimizing our system architecture to further reduce computation time and memory footprint.

\section{Appendix: edge extraction and sharpness index measure}

This section discusses our proposed method of extracting edges and measuring image sharpness. This is used to automatically identify the ground-truth from a given test image set, and for filtering unneeded images as input for the SR algorithm.

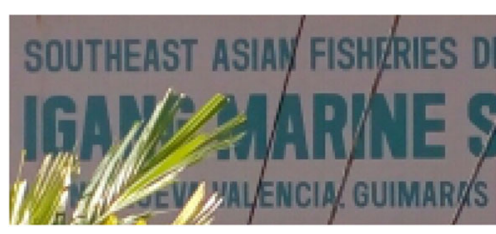

$\mathbf{a}$

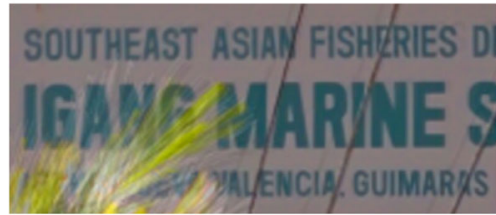

c

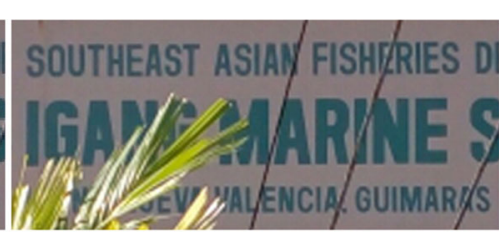

b

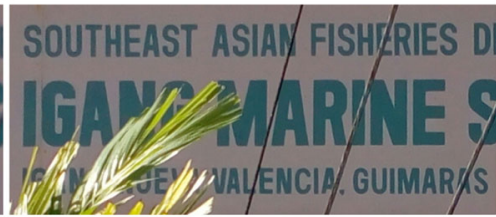

d

Fig. 19 Result for Structure_House. a Nearest-neighbor interpolation. b Bicubic interpolation. c Our SR method. d Ground-truth. Notice the ghosting effect caused by the moving leaves, which is a clear limitation of the mean fusion approach 

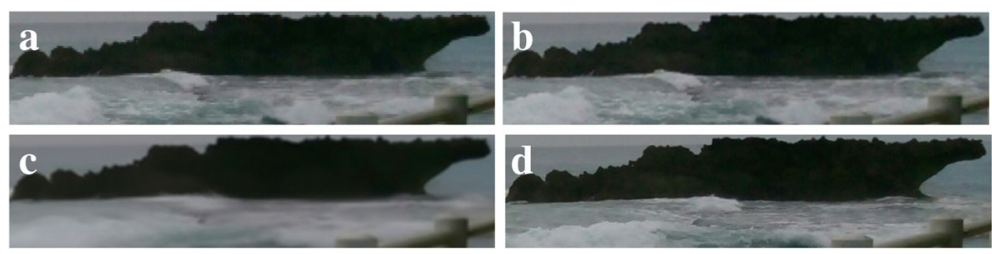

Fig. 20 Result for Scenery_Beach. a Nearest-neighbor interpolation. b Bicubic interpolation. c Our SR method. d Ground-truth. The textures and the edges of the rock were not fully recovered by the system. The movement of the waves caused unwanted ghosting effects

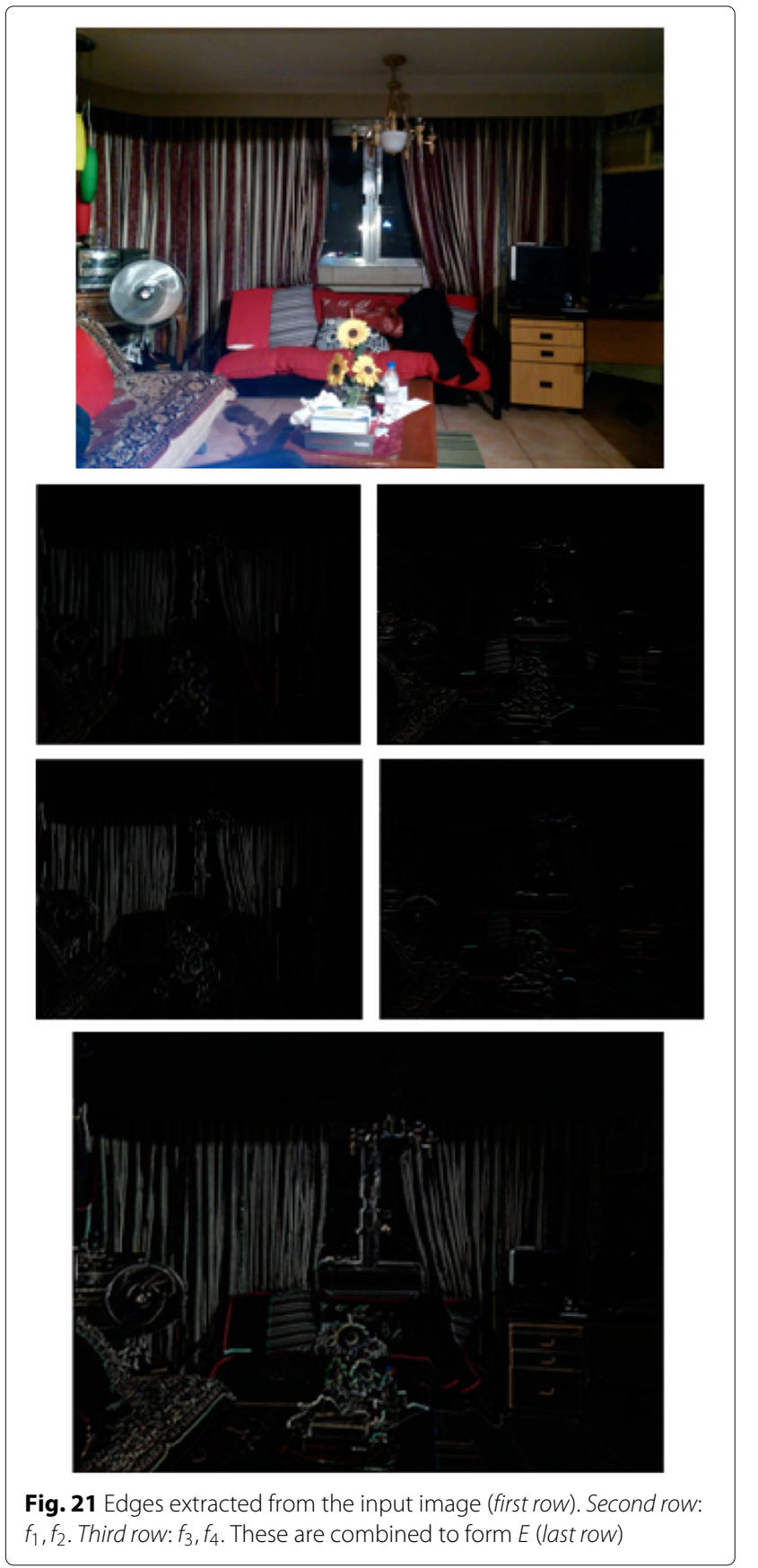

Given an image, $A$, to extract edges, we used the following filter kernels provided by [24].

$$
\begin{aligned}
& f_{1}=\left[\begin{array}{lll}
-101 & 0
\end{array} f_{2}=f_{1}{ }^{T}\right. \\
& f_{3}=[10-201] \quad f_{4}=f_{3}{ }^{T}
\end{aligned}
$$

Using the vector values provided in Eqs. 1 and 2, $f_{1}, f_{2}, f_{3}, f_{4}$ will be merged by mean fusion to form an aggregate of edges found in image $A$. Let $E$ be the aggregate of edges found in image $A$. The results should look like the sample images provided in Fig. 21.

Using $E$ with dimension of $H \times W$, calculating the sharpness index measure is performed by counting the nonzero elements of $E$ and divide it by $H \times W$. The sharpness index measure should have a range of $[0.0,1.0]$

\section{Authors' contributions}

The primary author, NDG, performed the study and implemented the prototype as guided by the adviser, Jl. Both authors read and approved the final manuscript.

\section{Competing interests}

The authors declare that they have no competing interests.

\section{Acknowledgements}

Both authors would like to acknowledge the Department of Science and Technology - Philippine Council for Industry, Energy and Emerging

Technology for funding this research.

Received: 1 June 2016 Accepted: 16 December 2016

Published online: 19 January 2017

\section{References}

1. D Mitzel, T Pock, T Schoenemann, D Cremers, in Proceedings of the 31st DAGM Symposium on Pattern Recognition. Video super resolution using duality based tv-11 optical flow (Springer, Berlin, Heidelberg, 2009), pp. 432-441

2. S Farsiu, MD Robinson, M Elad, P Milanfar, Fast and robust multiframe super resolution. IEEE Trans. Image Process. 13(10), 1327-1344 (2004)

3. C-H Chu, Super-resolution image reconstruction for mobile devices. Multimedia Syst. 19(4), 315-337 (2013)

4. J Simpkins, RL Stevenson, in Mathematical Optics: Classical, Quantum, and Computational. Methods Chapter No. 16. An introduction to super-resolution imaging (CRC Press, 2012)

5. R Timofte, V De Smet, L Van Gool, I Reid, H Saito, M-H Yang, in Computer Vision - ACCV 2014: 12th Asian Conference on Computer Vision. A+: Adjusted anchored neighborhood regression for fast super-resolution (Springer, Singapore, 2015), pp. 111-126

6. R Timofte, V De, LV Gool, in 2013 IEEE International Conference on Computer Vision. Anchored neighborhood regression for fast example-based super-resolution, (Sydney, 2013), pp. 1920-1927

7. A Nath, in 2013 International Conference on Communication Systems and Network Technologies (CSNT). Image denoising algorithms: A comparative 
study of different filtration approaches used in image restoration, (Gwalior, 2013), pp. 157-163

8. A Buades, B Coll, J-M Morel, Non-local means denoising. Image Processing Online. 1 (2011). http://dl.acm.org/citation.cfm?id=1069066

9. G Ghimpeteanu, T Batard, M Bertalmío, S Levine, A decomposition framework for image denoising algorithms. IEEE Trans. Image Process. 25(1), 388-399 (2016)

10. R Fergus, B Singh, A Hertzmann, ST Roweis, WT Freeman, in ACM SIGGRAPH 2006 Papers. SIGGRAPH '06. Removing camera shake from a single photograph (ACM, New York, NY, USA, 2006), pp. 787-794

11. L Yuan, I Sun, L Quan, H-Y Shum, in ACM SIGGRAPH 2007 Papers. SIGGRAPH '07. Image deblurring with blurred/noisy image pairs (ACM, New York, NY, USA, 2007)

12. X Chen, $X$ He, J Yang, Q Wu, in 2011 IEEE Conference on Computer Vision and Pattern Recognition (CVPR). An effective document image deblurring algorithm, (Colorado Springs, 2011), pp. 369-376

13. DP Mitchell, AN Netravali, in Proceedings of the 15th Annual Conference on Computer Graphics and Interactive Techniques. SIGGRAPH'88. Reconstruction filters in computer-graphics (ACM, New York, NY, USA, 1988), pp. 221-228

14. S Peleg, D Keren, L Schweitzer, Improving image resolution using subpixel motion. Pattern Recogn. Lett. 5(3), 223-226 (1987)

15. TS Huang, RY Tsay, in Advances in Computer Vision and Image Processing. Multiple frame image restoration and registration, vol. 1 (JAl, Greenwich, 1984), pp. 317-339

16. K Nasrollahi, TB Moeslund, Super-resolution: A comprehensive survey Mach. Vis. Appl. 25(6), 1423-1468 (2014)

17. T Yoshida, T Murakami, M Ikehara, in Intelligent Signal Processing and Communications Systems (ISPACS), 2013 International Symposium On. Image super-resolution method based on non-local means and self similarity, (2013), pp. 509-512. doi:10.1109/ISPACS.2013.6704604

18. G Farnebäck, in Proceedings of the 13th Scandinavian Conference on Image Analysis. SCIA'03. Two-frame motion estimation based on polynomial expansion (Springer, Berlin, Heidelberg, 2003), pp. 363-370

19. A Telea, An image inpainting technique based on the fast marching method. J. Graph. GPU, Game Tools. 9(1), 23-34 (2004)

20. WT Freeman, TR Jones, EC Pasztor, Example-based super-resolution. IEEE Comput. Graph. Appl. 22(2), 56-65 (2002)

21. D Glasner, S Bagon, M Irani, in International Conference on Computer Vision. Super-resolution from a single image, (Kyoto, 2009)

22. Q Shan, Z Li, J Jia, C-K Tang, Fast image/video upsampling. ACM Trans. Graph. 27(5), 153-11537 (2008)

23. C Mancas-Thillou, M Mirmehdi, An introduction to super-resolution text, 305-327 (2007). http://link.springer.com/chapter/10.1007\%2F978-184628-726-8_14\#page-1

24. J Yang, J Wright, TS Huang, Y Ma, Image super-resolution via sparse representation. IEEE Trans. Image Process. 19(11), 2861-2873 (2010)

25. M Protter, M Elad, H Takeda, P Milanfar, Generalizing the nonlocal-means to super-resolution reconstruction. IEEE Trans. Image Process. 18(1), 36-51 (2009)

26. E Rublee, V Rabaud, K Konolige, G Bradski, in Proceedings of the 2011 International Conference on Computer Vision. ICCV'11. Orb: An efficient alternative to sift or surf (IEEE Computer Society, Washington, DC, USA, 2011), pp. 2564-2571

27. D Capel, A Zisserman, Computer vision applied to super resolution. Signal Process. Mag. IEEE. 20(3), 75-86 (2003)

\section{Submit your manuscript to a SpringerOpen ${ }^{\circ}$ journal and benefit from:}

- Convenient online submission

- Rigorous peer review

- Immediate publication on acceptance

- Open access: articles freely available online

- High visibility within the field

Retaining the copyright to your article

Submit your next manuscript at $\gg$ springeropen.com 